九州大学学術情報リポジトリ

Kyushu University Institutional Repository

\title{
Morphological and Physiological Variation in Kakrol (Momordica dioica Roxb.)
}

\section{Rasul, Mohammad Golam}

Dept. of Genetics and Plant Breeding, BSMRAU

Hiramatsu, Michikazu

Laboratory of of Horticultural Science, Division of Agricultural Botany, Deaprtment of Plant Resources, Faculty of Agriculture, Kyushu University

Okubo, Hiroshi

Laboratory of of Horticultural Science, Division of Agricultural Botany, Deaprtment of Plant Resources, Faculty of Agriculture, Kyushu University

https://doi.org/10.5109/4559

出版情報: 九州大学大学院農学研究院紀要. 49 (1)，pp.1-11，2004-02-01. Faculty of Agriculture， Kyushu University

バージョン :

権利関係 : 


\title{
Morphological and Physiological Variation in Kakrol (Momordica dioica Roxb.)
}

\author{
Mohammad Golam RASUL ${ }^{1 *}$, Michikazu HIRAMATSU \\ and Hiroshi OKUBO \\ Laboratory of Horticultural Science, Division of Agricultural Botany, \\ Department of Plant Resources, Faculty of Agriculture, \\ Kyushu University, Fukuoka 812-8581, Japan \\ (Received October 10, 2003 and accepted November 14, 2003)
}

\begin{abstract}
Twenty nine kakrol (Momordica dioica Roxb.) accessions and its one wild relative ( $M$. cochinchinensis Spreng.), collected from different agro-ecological zones of Bangladesh, were evaluated for 29 morpho-physiological characters. Considerable variations were observed among the genotypes in which dissimilarity value ranged from 4.6 to 58.6. Cluster analysis based on this variation primarily classified dioica and chochinchinesis into two groups and formed five clusters in which one cluster contained 24 dioica accessions. No relationship was found between genetic divergence and geographical distribution of the accessions indicating no regional gene pool was responsible solely for clustering. Morpho-physiological variation is effective and hence proved its usefulness in classification of Momordica species. A kakrol descriptor is proposed which is to be used in characterization of kakrol cultivars or accessions.
\end{abstract}

\section{INTRODUCTION}

Kakrol (spine gourd, teasel gourd) belongs to family Cucurbitaceae having botanical name Momordica dioica Roxb. ( $2 \mathrm{n}=2 \mathrm{x}=28$, Trivedi and Roy, 1972). One of its wild relatives is $M$. cochinchinensis Spreng. ( $2 \mathrm{n}=4 \mathrm{x}=56$, Rashid, 1993) and it grows naturally in bush and jungle. $M$. cochinchinensis is characterized by larger leaves, flower and fruits than $M$. dioica. Both the species are dioecious and propagated vegetatively through tuberous roots.

Kakrol is a summer vegetable grown widely in Bangladesh. Now it has become a major vegetable in the country because of its high export potential and demand in internal market (Hossain et al., 1992). It is available in the market in the lean period of vegetables (April to October). It can stand handling well in transportation and has a good shelf life. It has good nutritional value (Bose and Som, 1986).

In spite of many advantages, there was no research thrust paid on kakrol's improvement. Genetic diversity or genetic relatedness and its utilization in breeding program based on morphological and physiological variation have been studied in many crops (Masud et al., 1995; Chowdhury et al., 1998; Rahman et al., 2001; Sultana et al., 2001; Grant et al., 2001; Brown and Myers, 2002). The knowledge on nature and magnitude of genetic variability existed in kakrol will provide the foundation of designing breeding program. Reports on genetic diversity, however, on morphological variation in kakrol are

\footnotetext{
1 Present address: Dept. of Genetics and Plant Breeding, BSMRAU, Salna, Gazipur 1706, Bangladesh
}

* Corresponding author (E-mail: rasul@bdonline.com) 
scanty.

The purpose of the present study was to keep a data base of morphological variability of collected plants, to assess the genetic diversity of local collections or landraces based on morphological and physiological variation, to evaluate their relationship and potential usefulness as genetic resources, to identify the relationship between geographical origin and genetic diversity of kakrol accessions and to propose a kakrol descriptor.

\section{MATERIALS AND METHODS}

\section{Plant materials}

Tuberous roots of 29 accessions of $M$. dioica Roxb. and one accession of $M$. cochinchinesis Spreng. were collected from different agro-ecological regions of Bangladesh in November 2001. They were maintained at the experimental farm of Bangabandhu Sheikh Mujibur Rahman Agricultural University (BSMRAU), Gazipur, Bangladesh.

\section{Planting time and cultural operations}

The experiment was conducted during March to September in the vinyl house of BSMRAU. The tuberous roots were planted in earthen pots containing a 1:1 mixture of soil and decomposed cowdung on February 8, 2002 and watered. They began to sprout 20-25 days after potting. The sprouted tuberous roots were transplanted in pits (30X $30 \times 30 \mathrm{~cm}$ ) in the experimental field on March 10,2002. The spacing between rows and between plants was $2.0 \mathrm{~m}$ and $1.5 \mathrm{~m}$, respectively. The male plants were planted in the field at 1:10 ratio (male : female).

The plants were supported by bamboo sticks. When the plants were about $1 \mathrm{~m}$ high, they were allowed to climb on rope net hanged vertically up to $2.5 \mathrm{~m}$ from the soil surface. Hand pollination was performed in the early morning by gently touching a stigma with the pollen of freshly dehisced anther. Fertilization and other cultivation techniques (intercultural operations) were followed as recommended by Hussain and Rashid (1974).

\section{Cluster analysis}

Twenty-nine parameters of morphological and physiological characteristics as listed in Table 1 were recorded from ten randomly selected plants. A data matrix was generated from the parameters of characteristics as listed in Table 1. Dissimilarity among the accessions was calculated using formula by Euclidean dissimilarity. Then a dendrogram was made by the Unweighted Pair-Group Methods using Arithmetic Average (UPGMA) (Sokal and Michener, 1958) using the software PHYLIP Version 3.57c (Felsenstein, 1995).

Table 1. Parameters (descriptor) of morphological and physiological characters studied in kakrol accessions.

\begin{tabular}{llll}
\hline \multicolumn{1}{c}{ Item } & \multicolumn{1}{c}{ Parameter } & \multicolumn{1}{c}{ Item } & \multicolumn{1}{c}{ Parameter } \\
\hline A Days to first & 1: Early (60-80 days) & O Style length & 1: Short $(<6 \mathrm{~mm})$ \\
flowering & 3: Medium early (81-100 days) & & 5: Medium $(6.1-9 \mathrm{~mm})$ \\
& 5: Medium (101-120 days) & & 9: Long $(>9.1 \mathrm{~mm})$ \\
& 7: Late (121-140 days) & P Pistil tip length & 1: Short $(<4 \mathrm{~mm})$ \\
\hline
\end{tabular}


Table 1. Continued.

\begin{tabular}{|c|c|c|c|}
\hline Item & Parameter & Item & Parameter \\
\hline $\begin{array}{l}\text { B No. of first flowering } \\
\text { node }\end{array}$ & $\begin{array}{l}1: 1-10 \text { nodes } \\
2: 11-20 \text { nodes } \\
3: 21-30 \text { nodes } \\
4: 31-40 \text { nodes } \\
5: 41-50 \text { nodes } \\
6: 51-60 \text { nodes } \\
7: 61-70 \text { nodes }\end{array}$ & Q Fruit color & $\begin{array}{l}\text { 5: Medium }(4.1-6.0 \mathrm{~mm}) \\
\text { 9: Long }(>6.1 \mathrm{~mm}) \\
\text { 1: Greenish yellow (GY) } \\
\text { 3: Yellow green (YG) } \\
\text { 5: Green (G) } \\
\text { 7: Dark green (DG) } \\
\text { 9: Light green orange (LG) }\end{array}$ \\
\hline C Stem color & $\begin{array}{l}\text { 1: Light green (LG) } \\
\text { 3: Green (G) } \\
\text { 5: Dark green (DG) }\end{array}$ & R Fruit shape & $\begin{array}{l}\text { 11: Light orange (LO) } \\
\text { 1: Round (R) } \\
\text { 3: Oval (Ov) }\end{array}$ \\
\hline D No. of ridge on stem & $\begin{array}{l}\text { 1: Present (5 ridge) } \\
9: \text { Absence (no ridge) }\end{array}$ & & $\begin{array}{l}\text { 5: Cylindrical }(\mathrm{C}) \\
\text { 7: Oblong (O) } \\
\text { 9: Spindle (S) }\end{array}$ \\
\hline $\begin{array}{l}\text { E Node color at the } \\
\text { attachment of the } \\
\text { leaves }\end{array}$ & $\begin{array}{l}\text { 1: Light green (LG) } \\
\text { 3: Green (G) } \\
\text { 5: Dark green (DG) } \\
\text { 7: Blackish (BL) }\end{array}$ & $\begin{array}{l}\text { S Conical spine } \\
\text { density }\end{array}$ & $\begin{array}{l}\text { 1: Thin (Tn) } \\
\text { 3: Thich (Tk) }\end{array}$ \\
\hline $\begin{array}{l}\text { F No. of stems per } \\
\text { plant }\end{array}$ & $\begin{array}{l}\text { 1: Few }(<10 \text { stems }) \\
\text { 5: Moderate }(11-20 \text { stems }) \\
\text { 9: Many }(>21 \text { stems })\end{array}$ & $\begin{array}{l}\text { T Conical spine } \\
\text { strength } \\
\text { U Pedicel attach- }\end{array}$ & $\begin{array}{l}\text { 1: Soft (S) } \\
\text { 3: Hard (H) } \\
\text { 1: Depressed (D) }\end{array}$ \\
\hline G Leaf color & $\begin{array}{l}\text { 1: Light green (LG) } \\
\text { 3: Green (G) } \\
\text { 5: Dark green (DG) }\end{array}$ & ment with the fruit & $\begin{array}{l}\text { 3: Slightly depressed (SD) } \\
\text { 5: Pointed (P) } \\
\text { 1: Short }(<50 \mathrm{~mm}) \\
\text { 3: Medium }(51-90 \mathrm{~mm})\end{array}$ \\
\hline H Leaf length & $\begin{array}{l}\text { 1: Short }(1-7 \mathrm{~cm}) \\
\text { 5: Medium }(7.1-15 \mathrm{~cm}) \\
\text { 9: Long }(>15.1 \mathrm{~cm})\end{array}$ & & $\begin{array}{l}\text { 5: Long }(91-130 \mathrm{~mm}) \\
\text { 7: Very long }(>131 \mathrm{~mm})\end{array}$ \\
\hline I Leaf width & $\begin{array}{l}\text { 1: Narrow }(1-7 \mathrm{~cm}) \\
\text { 5: Medium }(7.1-15 \mathrm{~cm}) \\
\text { 9: Wide }(>15.1 \mathrm{~cm})\end{array}$ & W Fruit diameter & $\begin{array}{l}\text { 1: Small }(1-40 \mathrm{~mm}) \\
\text { 5: Medium }(41-80 \mathrm{~mm}) \\
\text { 9: Large }(81-120 \mathrm{~mm})\end{array}$ \\
\hline $\mathbf{J}$ Leaf margin & $\begin{array}{l}\text { 1: Absence (no dentation) } \\
\text { 3: Slightly dented (SD) } \\
\text { 5: Medium dented (MD) } \\
\text { 7: Dentate (D) }\end{array}$ & $\begin{array}{l}\mathbf{X} \text { Single fruit } \\
\text { weight }\end{array}$ & $\begin{array}{l}\text { 1: Light }(1-50 \mathrm{~g}) \\
\text { 3: Medium }(51-100 \mathrm{~g}) \\
\text { 5: Heavy }(100-150 \mathrm{~g}) \\
\text { 7: Very heavy }(>151 \mathrm{~g})\end{array}$ \\
\hline $\mathbf{K}$ Leaf pubescence & $\begin{array}{l}\text { 9: Serrated (5 lobed) } \\
\text { 1: Absence (Ab) } \\
\text { 3: Few (F) } \\
\text { 5: Medium (M) }\end{array}$ & $\begin{array}{l}\text { Y No. of fruits per } \\
\text { plant }\end{array}$ & $\begin{array}{l}\text { 1: Few }(<20) \\
\text { 3: Moderate }(21-40) \\
\text { 5: Many }(41-60) \\
\text { 7: Profuse }(>61)\end{array}$ \\
\hline L Pedicel length & $\begin{array}{l}\text { 7: More }(\mathrm{Mo}) \\
\text { 1: Short }(1-7 \mathrm{~cm}) \\
\text { 5: Medium }(7.1-15 \mathrm{~cm}) \\
\text { 9: Long }(>15.1 \mathrm{~cm})\end{array}$ & $\begin{array}{l}\text { Z Fruit yield per } \\
\text { plant }\end{array}$ & $\begin{array}{l}\text { 1: Low }(<1.0 \mathrm{~kg}) \\
\text { 3: Medium }(1.1-2.0 \mathrm{~kg}) \\
\text { 5: Good }(2.1-3.0 \mathrm{~kg}) \\
\text { 7: Very good }(3.1-4.0) \\
\text { 9: Bumper }(>4.1 \mathrm{~kg}))\end{array}$ \\
\hline M Ovary length & $\begin{array}{l}\text { 1: Short }(1-10 \mathrm{~mm}) \\
\text { 5: Medium }(11-20 \mathrm{~mm}) \\
\text { 9: Long }(>21 \mathrm{~mm})\end{array}$ & $\begin{array}{l}\text { AA No. of seeds } \\
\text { per fruit }\end{array}$ & $\begin{array}{l}\text { 1: Few }(1-15) \\
\text { 3: Less }(16-30) \\
\text { 5: Medium }(31-45) \\
\text { 7: Many }(46-60)\end{array}$ \\
\hline $\mathbf{N}$ Ovary diameter & $\begin{array}{l}\text { 1: Small }(<7 \mathrm{~mm}) \\
\text { 5: Large }(>7.1 \mathrm{~mm})\end{array}$ & $\begin{array}{l}\text { AB 20-seed } \\
\text { weight }\end{array}$ & $\begin{array}{l}\text { 1: Light }(<2 \mathrm{~g}) \\
\text { 3: Medium }(2.1-4.0 \mathrm{~g}) \\
\text { 5: Slightly bold }(4.1-6.0 \mathrm{~g}) \\
\text { 7: Bold }(>6.1 \mathrm{~g})\end{array}$ \\
\hline
\end{tabular}




\section{RESULTS AND DISCUSSION}

\section{Days to first flowering and number of first flowering nodes}

Among 30 accessions, $63 \%$ showed early flowering habit (61 to 80 days), $33 \%$ had medium early and the rest single accession belonging to $M$. cochinchinensis was under late flowering group (121-140 days) (Table 2). Acc 2, Acc 3, Acc 4 and Acc 7 were noticeable for early flowering which produced flowers in 65, 58, 62 and 64 days after planting, respectively. Acc 3 produced its first flower at 9 th node of the plant while the wild accession, Acc 30 had it at 70th node and these two occupied two extreme groups. About $36 \%$ and $53 \%$ genotypes produced their flower within 11-20th and 21st-30th nodes, respectively.

Table 2. Categorization of morphological and physiological characteristics in kakrol accessions.

\begin{tabular}{|c|c|c|c|c|c|c|c|c|c|c|c|c|c|c|c|c|c|c|c|c|}
\hline \multirow{2}{*}{$\begin{array}{l}\text { Parameters } \\
\text { Accession } \\
\end{array}$} & \multicolumn{4}{|c|}{$\mathbf{A}^{\mathrm{a}}$} & \multicolumn{7}{|c|}{ B } & \multicolumn{3}{|c|}{ C } & \multicolumn{2}{|c|}{ D } & \multicolumn{4}{|c|}{$\mathbf{E}$} \\
\hline & $1^{\mathrm{a}}$ & 3 & 5 & 7 & 1 & 2 & 3 & 4 & 5 & 6 & 7 & 1 & 3 & 5 & 1 & 9 & 1 & 3 & 5 & 7 \\
\hline Acc 1 & $1^{\mathrm{b}}$ & $0^{\mathrm{b}}$ & 0 & 0 & 0 & 0 & 1 & 0 & 0 & 0 & 0 & 1 & 0 & 0 & 1 & 0 & 0 & 1 & 0 & 0 \\
\hline Acc 2 & 1 & 0 & 0 & 0 & 0 & 1 & 0 & 0 & 0 & 0 & 0 & 0 & 0 & 1 & 1 & 0 & 0 & 0 & 1 & 0 \\
\hline Acc 3 & 1 & 0 & 0 & 0 & 1 & 0 & 0 & 0 & 0 & 0 & 0 & 1 & 0 & 0 & 1 & 0 & 0 & 1 & 0 & 0 \\
\hline Acc 4 & 1 & 0 & 0 & 0 & 0 & 1 & 0 & 0 & 0 & 0 & 0 & 0 & 1 & 0 & 1 & 0 & 0 & 0 & 1 & 0 \\
\hline Acc 5 & 0 & 1 & 0 & 0 & 0 & 1 & 0 & 0 & 0 & 0 & 0 & 0 & 1 & 0 & 1 & 0 & 0 & 1 & 0 & 0 \\
\hline Acc 6 & 1 & 0 & 0 & 0 & 0 & 1 & 0 & 0 & 0 & 0 & 0 & 0 & 1 & 0 & 1 & 0 & 0 & 1 & 0 & 0 \\
\hline Acc 7 & 1 & 0 & 0 & 0 & 0 & 0 & 1 & 0 & 0 & 0 & 0 & 0 & 1 & 0 & 1 & 0 & 0 & 1 & 0 & 0 \\
\hline Acc 8 & 1 & 0 & 0 & 0 & 0 & 0 & 1 & 0 & 0 & 0 & 0 & 0 & 0 & 1 & 1 & 0 & 0 & 0 & 1 & 0 \\
\hline Acc 9 & 1 & 0 & 0 & 0 & 0 & 0 & 1 & 0 & 0 & 0 & 0 & 0 & 0 & 1 & 1 & 0 & 0 & 1 & 0 & 0 \\
\hline Acc 10 & 0 & 1 & 0 & 0 & 0 & 0 & 1 & 0 & 0 & 0 & 0 & 1 & 0 & 0 & 1 & 0 & 0 & 0 & 1 & 0 \\
\hline Acc 11 & 1 & 0 & 0 & 0 & 0 & 0 & 1 & 0 & 0 & 0 & 0 & 0 & 1 & 0 & 1 & 0 & 0 & 0 & 1 & 0 \\
\hline Acc 12 & 1 & 0 & 0 & 0 & 0 & 1 & 0 & 0 & 0 & 0 & 0 & 0 & 0 & 1 & 1 & 0 & 0 & 0 & 1 & 0 \\
\hline Acc 13 & 1 & 0 & 0 & 0 & 0 & 0 & 1 & 0 & 0 & 0 & 0 & 1 & 0 & 0 & 1 & 0 & 0 & 0 & 1 & 0 \\
\hline Acc 14 & 1 & 0 & 0 & 0 & 0 & 0 & 1 & 0 & 0 & 0 & 0 & 0 & 1 & 0 & 1 & 0 & 1 & 0 & 0 & 0 \\
\hline Acc 15 & 0 & 1 & 0 & 0 & 0 & 1 & 0 & 0 & 0 & 0 & 0 & 1 & 0 & 0 & 1 & 0 & 0 & 0 & 1 & 0 \\
\hline Acc 16 & 1 & 0 & 0 & 0 & 0 & 0 & 1 & 0 & 0 & 0 & 0 & 1 & 0 & 0 & 1 & 0 & 0 & 0 & 1 & 0 \\
\hline Acc 17 & 0 & 1 & 0 & 0 & 0 & 0 & 0 & 1 & 0 & 0 & 0 & 1 & 0 & 0 & 1 & 0 & 0 & 1 & 0 & 0 \\
\hline Acc 18 & 0 & 1 & 0 & 0 & 0 & 1 & 0 & 0 & 0 & 0 & 0 & 0 & 1 & 0 & 1 & 0 & 0 & 0 & 1 & 0 \\
\hline Acc 19 & 1 & 0 & 0 & 0 & 0 & 0 & 1 & 0 & 0 & 0 & 0 & 1 & 0 & 0 & 1 & 0 & 0 & 1 & 0 & 0 \\
\hline Acc 20 & 0 & 1 & 0 & 0 & 0 & 0 & 1 & 0 & 0 & 0 & 0 & 0 & 1 & 0 & 1 & 0 & 0 & 0 & 1 & 0 \\
\hline Acc 21 & 1 & 0 & 0 & 0 & 0 & 0 & 1 & 0 & 0 & 0 & 0 & 0 & 1 & 0 & 1 & 0 & 0 & 0 & 1 & 0 \\
\hline Acc 22 & 1 & 0 & 0 & 0 & 0 & 0 & 1 & 0 & 0 & 0 & 0 & 0 & 0 & 1 & 1 & 0 & 0 & 0 & 1 & 0 \\
\hline Acc 23 & 1 & 0 & 0 & 0 & 0 & 0 & 1 & 0 & 0 & 0 & 0 & 1 & 0 & 0 & 1 & 0 & 1 & 0 & 0 & 0 \\
\hline Acc 24 & 0 & 1 & 0 & 0 & 0 & 1 & 0 & 0 & 0 & 0 & 0 & 0 & 1 & 0 & 1 & 0 & 0 & 1 & 0 & 0 \\
\hline Acc 25 & 1 & 0 & 0 & 0 & 0 & 1 & 0 & 0 & 0 & 0 & 0 & 1 & 0 & 0 & 1 & 0 & 0 & 0 & 1 & 0 \\
\hline Acc 26 & 1 & 0 & 0 & 0 & 0 & 0 & 1 & 0 & 0 & 0 & 0 & 1 & 0 & 0 & 1 & 0 & 0 & 1 & 0 & 0 \\
\hline Acc 27 & 0 & 1 & 0 & 0 & 0 & 1 & 0 & 0 & 0 & 0 & 0 & 0 & 1 & 0 & 1 & 0 & 0 & 1 & 0 & 0 \\
\hline Acc 28 & 0 & 1 & 0 & 0 & 0 & 1 & 0 & 0 & 0 & 0 & 0 & 0 & 1 & 0 & 1 & 0 & 0 & 1 & 0 & 0 \\
\hline Acc 29 & 0 & 1 & 0 & 0 & 0 & 0 & 1 & 0 & 0 & 0 & 0 & 0 & 1 & 0 & 1 & 0 & 0 & 1 & 0 & 0 \\
\hline Acc 30 & 0 & 0 & 0 & 1 & 0 & 0 & 0 & 0 & 0 & 0 & 1 & 0 & 0 & 1 & 0 & 1 & 0 & 0 & 1 & 0 \\
\hline Total & 19 & 10 & 0 & 1 & 1 & 11 & 16 & 1 & 0 & 0 & 1 & 11 & 13 & 6 & 29 & 1 & 2 & 13 & 15 & 0 \\
\hline
\end{tabular}

a: Explanation of $\mathbf{A}$ to $\mathbf{A B}$ and number $\mathbf{1}$ to $\mathbf{1 1}$ is described in Table 1.

b: 1 and 0 represent the presence and absence of the accessions in that category, respectively. 
Table 2. Continued.

\begin{tabular}{|c|c|c|c|c|c|c|c|c|c|c|c|c|c|c|c|c|c|c|c|c|c|}
\hline \multirow{2}{*}{$\begin{array}{l}\text { Parameters } \\
\text { Accession } \\
\end{array}$} & \multicolumn{3}{|c|}{$\mathbf{F}$} & \multicolumn{3}{|c|}{ G } & \multicolumn{3}{|c|}{$\mathbf{H}$} & \multicolumn{3}{|c|}{ I } & \multicolumn{5}{|c|}{$\mathbf{J}$} & \multicolumn{4}{|c|}{$\mathbf{K}$} \\
\hline & 1 & 5 & 9 & 1 & 3 & 5 & 1 & 5 & 9 & 1 & 5 & 9 & 1 & 3 & 5 & 7 & 9 & 1 & 3 & 5 & 7 \\
\hline Acc 1 & 0 & 1 & 0 & 0 & 1 & 0 & 0 & 1 & 0 & 0 & 1 & 0 & 0 & 0 & 1 & 0 & 0 & 0 & 1 & 0 & 0 \\
\hline Acc 2 & 0 & 1 & 0 & 0 & 0 & 1 & 0 & 1 & 0 & 0 & 1 & 0 & 0 & 0 & 1 & 0 & 0 & 0 & 0 & 1 & 0 \\
\hline Acc 3 & 0 & 1 & 0 & 0 & 1 & 0 & 0 & 1 & 0 & 0 & 1 & 0 & 0 & 1 & 0 & 0 & 0 & 0 & 1 & 0 & 0 \\
\hline $\operatorname{Acc} 4$ & 0 & 1 & 0 & 0 & 1 & 0 & 0 & 1 & 0 & 0 & 1 & 0 & 0 & 0 & 1 & 0 & 0 & 0 & 1 & 0 & 0 \\
\hline Acc 5 & 0 & 1 & 0 & 0 & 1 & 0 & 0 & 1 & 0 & 0 & 1 & 0 & 0 & 0 & 0 & 1 & 0 & 0 & 0 & 1 & 0 \\
\hline Acc 6 & 0 & 1 & 0 & 0 & 1 & 0 & 0 & 1 & 0 & 0 & 1 & 0 & 0 & 1 & 0 & 0 & 0 & 0 & 1 & 0 & 0 \\
\hline Acc 7 & 0 & 1 & 0 & 0 & 0 & 1 & 0 & 1 & 0 & 0 & 1 & 0 & 0 & 1 & 0 & 0 & 0 & 0 & 1 & 0 & 0 \\
\hline Acc 8 & 0 & 1 & 0 & 0 & 0 & 1 & 0 & 1 & 0 & 0 & 1 & 0 & 0 & 1 & 0 & 0 & . & 0 & 1 & 0 & 0 \\
\hline Acc 9 & 0 & 1 & 0 & 0 & 0 & 1 & 0 & 1 & 0 & 0 & 1 & 0 & 0 & 0 & 0 & 1 & 0 & 0 & 1 & 0 & 0 \\
\hline Acc 10 & 0 & 0 & 1 & 0 & 1 & 0 & 0 & 1 & 0 & 0 & 1 & 0 & 0 & 0 & 0 & 1 & 0 & 0 & 1 & 0 & 0 \\
\hline Acc 11 & 0 & 1 & 0 & 0 & 0 & 1 & 0 & 1 & 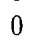 & 0 & 1 & 0 & 0 & 0 & 1 & 0 & 0 & 0 & 0 & 1 & 0 \\
\hline Acc 12 & 0 & 1 & 0 & 1 & 0 & 0 & 0 & 1 & 0 & 0 & 1 & 0 & 0 & 0 & 0 & 1 & 0 & 0 & 0 & 1 & 0 \\
\hline Acc 13 & 0 & 1 & 0 & 0 & 0 & 1 & 0 & 1 & 0 & 0 & 1 & 0 & 0 & 1 & 0 & 0 & . & 0 & 1 & 0 & 0 \\
\hline Acc 14 & 0 & 1 & 0 & 0 & 1 & 0 & 0 & 1 & 0 & 0 & 1 & 0 & 0 & 0 & 1 & 0 & 0 & 0 & 1 & 0 & 0 \\
\hline Acc 15 & 0 & 1 & 0 & 1 & 0 & 0 & 0 & 1 & 0 & 0 & 1 & 0 & 0 & 0 & 0 & 1 & 0 & 0 & 1 & 0 & 0 \\
\hline Acc 16 & 0 & 0 & 1 & 0 & 1 & 0 & 0 & 1 & 0 & 0 & 1 & 0 & 0 & 0 & 1 & 0 & 0 & 0 & 1 & 0 & 0 \\
\hline Acc 17 & 0 & 1 & 0 & 0 & 1 & 0 & 0 & 1 & 0 & 0 & 1 & 0 & 0 & 0 & 0 & 1 & 0 & 0 & 1 & 0 & 0 \\
\hline Acc 18 & 0 & 1 & 0 & 0 & 1 & 0 & 0 & 1 & 0 & 0 & 1 & 0 & 0 & 0 & 0 & 1 & 0 & 0 & 1 & 0 & 0 \\
\hline Acc 19 & 0 & 1 & 0 & 0 & 1 & 0 & 0 & 1 & 0 & 0 & 1 & 0 & 0 & 1 & 0 & 0 & 0 & 0 & 1 & 0 & 0 \\
\hline Acc 20 & 0 & 1 & 0 & 0 & 1 & 0 & 0 & 1 & 0 & 0 & 1 & 0 & 0 & 0 & 1 & 0 & 0 & 0 & 1 & 0 & 0 \\
\hline Acc 21 & 0 & 0 & 1 & 0 & 1 & 0 & 0 & 1 & 0 & 0 & 1 & 0 & 0 & 0 & 1 & 0 & 0 & 0 & 1 & 0 & 0 \\
\hline Acc 22 & 0 & 1 & 0 & 0 & 0 & 1 & 0 & 1 & 0 & 0 & 1 & 0 & 0 & 1 & 0 & 0 & 0 & 0 & 1 & 0 & 0 \\
\hline Acc 23 & 0 & 0 & 1 & 0 & 1 & 0 & 0 & 1 & 0 & 0 & 1 & 0 & 0 & 1 & 0 & 0 & 0 & 0 & 1 & 0 & 0 \\
\hline Acc 24 & 0 & 1 & 0 & 0 & 1 & 0 & 0 & 1 & 0 & 0 & 1 & 0 & 0 & 0 & 0 & 1 & 0 & 0 & 1 & 0 & 0 \\
\hline Acc 25 & 0 & 1 & 0 & 0 & 1 & 0 & 0 & 1 & 0 & 0 & 1 & 0 & 0 & 1 & 0 & 0 & 0 & 0 & 0 & 1 & 0 \\
\hline Acc 26 & 0 & 0 & 1 & 0 & 0 & 1 & 0 & 1 & 0 & 0 & 1 & 0 & 0 & 0 & 1 & 0 & 0 & 0 & 1 & 0 & 0 \\
\hline Acc 27 & 0 & 0 & 1 & 0 & 0 & 1 & 0 & 1 & 0 & 0 & 1 & 0 & 0 & 1 & 0 & 0 & 0 & 0 & 1 & 0 & 0 \\
\hline $\operatorname{Acc} 28$ & 0 & 1 & 0 & 0 & 1 & 0 & 0 & 1 & 0 & 0 & 1 & 0 & 0 & 0 & 1 & 0 & 0 & 0 & 1 & 0 & 0 \\
\hline Acc 29 & 0 & 1 & 0 & 0 & 1 & 0 & 0 & 1 & 0 & 0 & 1 & 0 & 0 & 0 & 1 & 0 & 0 & 0 & 1 & 0 & 0 \\
\hline Acc 30 & 1 & 0 & 0 & 0 & 0 & 1 & 0 & 0 & 1 & 0 & 0 & 1 & 0 & 0 & 0 & 0 & 1 & 1 & 0 & 0 & 0 \\
\hline Total & 1 & 23 & 6 & 2 & 18 & 10 & 0 & 29 & 1 & 0 & 29 & 1 & 0 & 10 & 11 & 8 & 1 & 1 & 24 & 5 & 0 \\
\hline
\end{tabular}

\section{Stem characteristics}

Stem color was grouped into light green (36\%), green (43\%) and dark green (20\%) (Table 2). All the $M$. dioica accessions possessed five ridges on their stem while no ridge rather round shaped stem was exhibited in $M$. cochinchinensis and this dark green round shaped stem might be a distinction feature between these two species. The color of the nodes at attachment of the leaves was found light green to dark green. The number of stems per plant was graded as few, moderate and many. The number was counted in the plant at $30 \mathrm{~cm}$ height above the ground. About $76 \%$ genotypes had moderate (11-20 stems) number of stems, $20 \%$ had many (more than 20 ) and $3 \%$ (only Acc 30 ) had few (only three) stems. Number of stems per plant was found effective to discriminate dioica from cochinchinensis species but not efficient within dioica accessions.

\section{Leaf characteristics}

The leaves were characterized by its color, length, width, margin and pubescence. 
Table 2. Continued.

\begin{tabular}{|c|c|c|c|c|c|c|c|c|c|c|c|c|c|c|c|c|c|c|c|c|}
\hline Parameters & \multicolumn{3}{|c|}{$\mathbf{L}$} & \multicolumn{3}{|c|}{$\mathbf{M}$} & \multicolumn{2}{|c|}{$\mathbf{N}$} & \multicolumn{3}{|c|}{$\mathbf{0}$} & \multicolumn{3}{|c|}{$\mathbf{P}$} & \multicolumn{4}{|c|}{$\mathbf{Q}$} & \multirow[b]{2}{*}{9} & \multirow[b]{2}{*}{11} \\
\hline Accession & 1 & 5 & 9 & 1 & 5 & 9 & 1 & 5 & 1 & 5 & 9 & 1 & 5 & 9 & 1 & 3 & 5 & 7 & & \\
\hline Acc 1 & 0 & 1 & 0 & 0 & 1 & 0 & 1 & 0 & 0 & 1 & 0 & 0 & 1 & 0 & 0 & 1 & 0 & 0 & 0 & 0 \\
\hline Acc 2 & 0 & 1 & 0 & 0 & 1 & 0 & 1 & 0 & 0 & 1 & 0 & 0 & 1 & 0 & 1 & 0 & 0 & 0 & 0 & 0 \\
\hline Acc 3 & 0 & 1 & 0 & 0 & 1 & 0 & 1 & 0 & 0 & 1 & 0 & 0 & 1 & 0 & 0 & 1 & 0 & 0 & 0 & 0 \\
\hline Acc 4 & 0 & 1 & 0 & 0 & 1 & 0 & 1 & 0 & 0 & 1 & 0 & 0 & 1 & 0 & 1 & 0 & 0 & 0 & 0 & 0 \\
\hline Acc 5 & 0 & 1 & 0 & 0 & 1 & 0 & 0 & 1 & 0 & 0 & 1 & 0 & 1 & 0 & 1 & 0 & 0 & 0 & 0 & 0 \\
\hline $\operatorname{Acc} 6$ & 0 & 0 & 1 & 0 & 1 & 0 & 1 & 0 & 0 & 1 & 0 & 0 & 1 & 0 & 0 & 0 & 0 & 1 & 0 & 0 \\
\hline Acc 7 & 0 & 0 & 1 & 0 & 1 & 0 & 0 & 1 & 0 & 1 & 0 & 0 & 1 & 0 & 0 & 0 & 0 & 1 & 0 & 0 \\
\hline Acc 8 & 0 & 0 & 1 & 0 & 1 . & 0 & 0 & 1 & 0 & 1 & 0 & 0 & 1 & 0 & 0 & 0 & 0 & 1 & 0 & 0 \\
\hline Acc 9 & 0 & 1 & 0 & 0 & 1 & 0 & 0 & 1 & 0 & 1 & 0 & 0 & 1 & 0 & 0 & 0 & 0 & 0 & 1 & 0 \\
\hline Acc 10 & 0 & 0 & 1 & 0 & 1 & 0 & 1 & 0 & 0 & 1 & 0 & 0 & 1 & 0 & 0 & 1 & 0 & 0 & 0 & 0 \\
\hline Acc 11 & 0 & 0 & 1 & 0 & 1 & 0 & 0 & 1 & 0 & 1 & 0 & 0 & 1 & 0 & 1 & 0 & 0 & 0 & 0 & 0 \\
\hline Acc 12 & 0 & 1 & 0 & 0 & 1 & 0 & 1 & 0 & 0 & 1 & 0 & 0 & 1 & 0 & 0 & 0 & 0 & 1 & 0 & 0 \\
\hline Acc 13 & 0 & 1 & 0 & 0 & 1 & 0 & 1 & 0 & 0 & 0 & 1 & 0 & 1 & 0 & 1 & 0 & 0 & 0 & 0 & 0 \\
\hline Acc 14 & 0 & 0 & 1 & 0 & 1 & 0 & 0 & 1 & 0 & 1 & 0 & 0 & 1 & 0 & 1 & 0 & 0 & 0 & 0 & 0 \\
\hline Acc 15 & 0 & 1 & 0 & 0 & 1 & 0 & 0 & 1 & 0 & 1 & 0 & 0 & 1 & 0 & 0 & 1 & 0 & 0 & 0 & 0 \\
\hline Acc 16 & 0 & 1 & 0 & 0 & 1 & 0 & 1 & 0 & 0 & 1 & 0 & 0 & 1 & 0 & 0 & 1 & 0 & 0 & 0 & 0 \\
\hline Acc 17 & 0 & 1 & 0 & 0 & 1 & 0 & 1 & 0 & 0 & 1 & 0 & 0 & 1 & 0 & 1 & 0 & 0 & 0 & 0 & 0 \\
\hline Acc 18 & 0 & 1 & 0 & 0 & 1 & 0 & 1 & 0 & 0 & 1 & 0 & 0 & 1 & 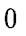 & 0 & 1 & 0 & 0 & 0 & 0 \\
\hline Acc 19 & 0 & 1 & 0 & 0 & 1 & 0 & 1 & 0 & 0 & 1 & 0 & 0 & 1 & 0 & 0 & 0 & 1 & 0 & 0 & 0 \\
\hline Acc 20 & 0 & 1 & 0 & 0 & 1 & 0 & 1 & 0 & 0 & 1 & 0 & 0 & 1 & 0 & 1 & 0 & 0 & 0 & 0 & 0 \\
\hline Acc 21 & 0 & 0 & 1 & 0 & 1 & 0 & 0 & 1 & 0 & 0 & 1 & 0 & 1 & 0 & 1 & 0 & 0 & 0 & 0 & 0 \\
\hline Acc 22 & 0 & 0 & 1 & 0 & 1 & 0 & 1 & 0 & 0 & 1 & 0 & 0 & 1 & 0 & 0 & 1 & 0 & 0 & 0 & 0 \\
\hline $\operatorname{Acc} 23$ & 0 & 1 & 0 & 0 & 0 & 1 & 0 & 1 & 0 & 1 & 0 & 0 & 1 & 0 & 0 & 0 & 1 & 0 & 0 & 0 \\
\hline $\operatorname{Acc} 24$ & 0 & 0 & 1 & 0 & 1 & 0 & 0 & 1 & 0 & 1 & 0 & 0 & 1 & 0 & 0 & 1 & 0 & 0 & 0 & 0 \\
\hline $\operatorname{Acc} 25$ & 0 & 1 & 0 & 0 & 1 & 0 & 1 & 0 & 0 & 1 & 0 & 0 & 1 & 0 & 0 & 1 & 0 & 0 & 0 & 0 \\
\hline $\operatorname{Acc} 26$ & 0 & 1 & 0 & 0 & 1 & 0 & 0 & 1 & 0 & 1 & 0 & 0 & 1 & 0 & 1 & 0 & 0 & 0 & 0 & 0 \\
\hline $\operatorname{Acc} 27$ & 0 & 1 & 0 & 0 & 1 & 0 & 1 & 0 & 0 & 1 & 0 & 0 & 1 & 0 & 0 & 1 & 0 & 0 & 0 & 0 \\
\hline $\operatorname{Acc} 28$ & 0 & 1 & 0 & 0 & 1 & 0 & 1 & 0 & 0 & 1 & 0 & 0 & 1 & 0 & 1 & 0 & 0 & 0 & 0 & 0 \\
\hline Acc 29 & 0 & 1 & 0 & 0 & 1 & 0 & 0 & 1 & 0 & 1 & 0 & 0 & 1 & 0 & 0 & 1 & 0 & 0 & 0 & 0 \\
\hline Acc 30 & 0 & 1 & 0 & 0 & 0 & 1 & 0 & 1 & 0 & 1 & 0 & 1 & 0 & 0 & 0 & 0 & 0 & 1 & 0 & 0 \\
\hline Total & 0 & 21 & 9 & 0 & 28 & 2 & 17 & 13 & 0 & 27 & 3 & 1 & 29 & 0 & 11 & 11 & 2 & 5 & 1 & 0 \\
\hline
\end{tabular}

These were measured in full growth stage of the plant: About $60 \%$ and $33 \%$ accessions had green and dark green leaves, respectively. Only two genotypes (Acc 12 and Acc 15) showed light green leaves. Leaf length and width showed similar trend in grouping the genotypes. All the accessions except Acc 30 had medium leaves. Acc 30 exhibited with long and wide leaves. Leaf margin may be used as distinguishing feature between dioica and cochinchinensis, the former is dented (small serration present all along the leaf edge) and the later is serrated (lobe is finger like). M. cochinchinensis (Acc 30) possessed serrated leaves having five deep lobe while other dioica accessions, viz. 33, 37 and $27 \%$ accessions had slightly dented, medium dented and dented leaves, respectively. About 94\% genotypes had few to medium pubescence present on leaf surface while it was completely absent in $M$. cochinchinensis (Acc 30). It might be another morphological marker of distinguishing these two species. 
Table 2. Continued.

\begin{tabular}{|c|c|c|c|c|c|c|c|c|c|c|c|c|c|c|c|c|c|c|c|}
\hline Parameters & \multicolumn{5}{|c|}{$\mathbf{R}$} & \multicolumn{2}{|c|}{$\mathbf{S}$} & \multicolumn{2}{|c|}{$\mathbf{T}$} & \multicolumn{3}{|c|}{$\mathbf{U}$} & \multicolumn{4}{|c|}{$\mathrm{V}$} & \multicolumn{3}{|c|}{$\mathbf{W}$} \\
\hline Accession & 1 & 3 & 5 & 7 & 9 & 1 & 3 & 1 & 3 & 1 & 3 & 5 & 1 & 3 & 5 & 7 & 1 & 5 & 9 \\
\hline Acc 1 & 0 & 1 & 0 & 0 & 0 & 0 & 1 & 1 & 0 & 0 & 0 & 1 & 0 & 1 & 0 & 0 & 0 & 1 & 0 \\
\hline Acc 2 & 0 & 1 & 0 & 0 & 0 & 0 & 1 & 1 & 0 & 0 & 1 & 0 & 0 & 1 & 0 & 0 & 0 & 1 & 0 \\
\hline Acc 3 & 0 & 1 & 0 & 0 & 0 & 0 & 1 & 1 & 0 & 1 & 0 & 0 & 0 & 1 & 0 & 0 & 0 & 1 & 0 \\
\hline Acc 4 & 0 & 0 & 0 & 1 & 0 & 0 & 1 & 1 & 0 & 0 & 0 & 1 & 0 & 1 & 0 & 0 & 0 & 1 & 0 \\
\hline $\operatorname{Acc} 5$ & 0 & 0 & 0 & 0 & 1 & 0 & 1 & 1 & 0 & 0 & 0 & 1 & 0 & 1 & 0 & 0 & 0 & 1 & 0 \\
\hline Acc 6 & 0 & 0 & 1 & 0 & 0 & 1 & 0 & 0 & 1 & 0 & 1 & 0 & 0 & 1 & 0 & 0 & 0 & 1 & 0 \\
\hline $\operatorname{Acc} 7$ & 0 & 0 & 1 & 0 & 0 & 1 & 0 & 0 & 1 & 0 & 1 & 0 & 0 & 1 & 0 & 0 & 0 & 1 & 0 \\
\hline Acc 8 & 0 & 0 & 1 & 0 & 0 & 0 & 1 & 0 & 1 & 0 & 1 & 0 & 0 & 0 & 1 & 0 & 0 & 1 & 0 \\
\hline Acc 9 & 0 & 0 & 1 & 0 & 0 & 0 & 1 & 0 & 1 & 0 & 0 & 1 & 0 & 0 & 1 & 0 & 0 & 1 & 0 \\
\hline Acc 10 & 0 & 0 & 1 & 0 & 0 & 0 & 1 & 0 & 1 & 0 & 1 & 0 & 0 & 1 & 0 & 0 & 0 & 1 & 0 \\
\hline Acc 11 & 0 & 1 & 0 & 0 & 0 & 0 & 1 & 0 & 1 & 0 & 1 & 0 & 0 & 1 & 1 & 0 & 0 & 1 & 0 \\
\hline Acc 12 & 0 & 0 & 0 & 0 & 1 & 1 & 0 & 0 & 1 & 0 & 0 & 1 & 0 & 0 & 0 & 0 & 0 & 1 & 0 \\
\hline Acc 13 & 0 & 1 & 0 & 0 & 0 & 0 & 1 & 0 & 1 & 1 & 0 & 0 & 0 & 1 & 0 & 0 & 0 & 1 & 0 \\
\hline Acc 14 & 0 & 0 & 0 & 1 & 0 & 0 & 1 & 1 & 0 & 0 & 1 & 0 & 0 & 1 & 0 & 0 & 0 & 1 & 0 \\
\hline Acc 15 & 0 & 0 & 0 & 1 & 0 & 0 & 1 & 1 & 0 & 0 & 0 & 1 & 0 & 1 & 0 & 0 & 1 & 0 & 0 \\
\hline Acc 16 & 0 & 0 & 0 & 1 & 0 & 0 & 1 & 0 & 1 & 0 & 1 & 0 & 0 & 1 & 0 & 0 & 0 & 1 & 0 \\
\hline Acc 17 & 0 & 0 & 1 & 0 & 0 & 0 & 1 & 0 & 1 & 0 & 1 & 0 & 0 & 1 & 0 & 0 & 0 & 1 & 0 \\
\hline Acc 18 & 0 & 0 & 0 & 1 & 0 & 0 & 1 & 0 & 1 & 0 & 1 & 0 & 0 & 1 & 0 & 0 & 0 & 1 & 0 \\
\hline Acc 19 & 0 & 1 & 0 & 0 & 0 & 0 & 1 & 0 & 1 & 0 & 1 & 0 & 0 & 0 & 1 & 0 & 0 & 1 & 0 \\
\hline $\operatorname{Acc} 20$ & 0 & 1 & 0 & 0 & 0 & 0 & 1 & 0 & 1 & 0 & 1 & 0 & 0 & 1 & 0 & 0 & 0 & 1 & 0 \\
\hline Acc 21 & 0 & 0 & 0 & 1 & 0 & 0 & 1 & 0 & 1 & 0 & 0 & 1 & 0 & 1 & 0 & 0 & 0 & 1 & 0 \\
\hline Acc 22 & 0 & 0 & 0 & 1 & 0 & 0 & 1 & 0 & 1 & 0 & 0 & 1 & 0 & 1 & 0 & 0 & 0 & 1 & 0 \\
\hline Acc 23 & 0 & 1 & 0 & 0 & 0 & 0 & 1 & 0 & 1 & 0 & 0 & 1 & 0 & 1 & 0 & 0 & 0 & 1 & 0 \\
\hline $\operatorname{Acc} 24$ & 0 & 0 & 0 & 0 & 1 & 1 & 0 & 0 & 1 & 0 & 0 & 1 & 0 & 1 & 0 & 0 & 1 & 0 & 0 \\
\hline Acc 25 & 0 & 1 & 0 & 0 & 0 & 0 & 1 & 1 & 0 & 0 & 1 & 0 & 0 & 1 & 0 & 0 & 0 & 1 & 0 \\
\hline Acc 26 & 0 & 0 & 0 & 0 & 1 & 0 & 1 & 0 & 1 & 1 & 0 & 0 & 0 & 1 & 0 & 0 & 0 & 1 & 0 \\
\hline Acc 27 & 0 & 1 & 0 & 0 & 0 & 0 & 1 & 0 & 1 & 0 & 1 & 0 & 0 & 1 & 0 & 0 & 0 & 1 & 0 \\
\hline Acc 28 & 0 & 1 & 0 & 0 & 0 & 0 & 1 & 0 & 1 & 0 & 1 & 0 & 0 & 1 & 0 & 0 & 0 & 1 & 0 \\
\hline Acc 29 & 1 & 0 & 0 & 0 & 0 & 0 & 1 & 1 & 0 & 0 & 0 & 1 & 1 & 0 & 0 & 0 & 1 & 0 & 0 \\
\hline Acc 30 & 0 & 0 & 0 & 0 & 1 & 1 & 0 & 0 & 1 & 0 & 0 & 1 & 0 & 0 & 0 & 1 & 0 & 0 & 1 \\
\hline Total & 1 & 11 & 6 & 7 & 5 & 5 & 25 & 9 & 21 & 3 & 15 & 12 & 1 & 24 & 4 & 1 & 3 & 26 & 1 \\
\hline
\end{tabular}

\section{Flower characteristics}

There were no differences observed in green sepal and petal colors. Flower characteristics included pedicel length, ovary length and diameter, style length and pistil tip length. Pedicel length was divided into the category of short (0\%), medium (70\%) and long (30\%). The longer the pedicel length, the easier to pick the fruits up during harvest. Fruits with short pedicel usually are hidden under the leaves and cause problems during harvest (generally fruits are hanged under the net). About $57 \%$ accessions had small ovary diameter and others (43\%) contained large diameter. It seemed that ovary size has positive correlation with fruit size. Almost all the genotypes showed medium style and pistil tip length with the exception of Acc 30 that had short pistil tip (less than $4 \mathrm{~mm}$ ) while Acc 5, Acc 13 and Acc 21 had long style length.

\section{Fruit characteristics}

The color of fruits during edible maturity showed great variability among the 
Table 2. Continued.

\begin{tabular}{|c|c|c|c|c|c|c|c|c|c|c|c|c|c|c|c|c|c|c|c|c|c|}
\hline \multirow{2}{*}{$\begin{array}{l}\text { Parameters } \\
\text { Accession } \\
\end{array}$} & \multicolumn{4}{|c|}{$\mathrm{X}$} & \multicolumn{4}{|c|}{$Y$} & \multicolumn{5}{|c|}{$\mathbf{Z}$} & \multicolumn{4}{|c|}{$\mathbf{A A}$} & \multicolumn{4}{|c|}{$\mathbf{A B}$} \\
\hline & 1 & 3 & 5 & 7 & 1 & 3 & 5 & 7 & 1 & 3 & 5 & 7 & 9 & 1 & 3 & 5 & 7 & 1 & 3 & 5 & 7 \\
\hline Acc 1 & 0 & 1 & 0 & 0 & 0 & 0 & 1 & 0 & 0 & 0 & 1 & 0 & 0 & 0 & 1 & 0 & 0 & 0 & 0 & 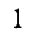 & 0 \\
\hline Acc 2 & 0 & 1 & 0 & 0 & 0 & 1 & 0 & 0 & 0 & 0 & 1 & 0 & 0 & 0 & 1 & 0 & 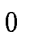 & 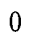 & 1 & 0 & \\
\hline Acc 3 & 1 & 0 & 0 & 0 & 0 & 0 & 0 & 1 & 0 & 0 & 1 & 0 & 0 & 0 & 1 & 0 & 0 & 0 & 1 & 0 & \\
\hline Acc 4 & 0 & 0 & 1 & 0 & 0 & 0 & 1 & 0 & 0 & 0 & 0 & 0 & 0 & 0 & 1 & 0 & 0 & 0 & 0 & 1 & \\
\hline Acc 5 & 0 & 1 & 0 & 0 & 0 & 1 & 0 & 0 & 0 & 1 & 0 & 0 & 1 & 0 & 0 & 1 & 0 & 0 & 0 & 1 & \\
\hline Acc 6 & 0 & 1 & 0 & 0 & 0 & 0 & 1 & 0 & 0 & 0 & 1 & 0 & 0 & 0 & 0 & 1 & 0 & 0 & 0 & 1 & 0 \\
\hline Acc 7 & 0 & 1 & 0 & 0 & 0 & 0 & 0 & 1 & 0 & 0 & 0 & 0 & 1 & 0 & 0 & 1 & 0 & 0 & 0 & 1 & 0 \\
\hline $\operatorname{Acc} 8$ & 0 & 0 & 1 & 0 & 0 & 1 & 0 & 0 & 0 & 0 & 0 & 0 & 1 & 0 & 0 & 0 & 1 & 0 & 1 & 0 & 0 \\
\hline Acc 9 & 0 & 0 & 1 & 0 & 0 & 1 & 0 & 0 & 0 & 0 & 0 & 0 & 1 & 0 & 0 & 1 & 0 & 0 & 0 & 1 & 0 \\
\hline Acc 10 & 0 & 1 & 0 & 0 & 0 & 1 & 0 & 0 & 0 & 0 & 0 & 1 & 0 & 0 & 0 & 1 & 0 & 0 & 0 & 1 & 0 \\
\hline Acc 11 & 0 & 1 & 0 & 0 & 1 & 0 & 0 & 0 & 0 & 1 & 0 & 0 & 0 & 0 & 1 & 0 & 0 & 0 & 0 & 1 & 0 \\
\hline Acc 12 & 1 & 0 & 0 & 0 & 0 & 0 & 1 & 0 & 0 & 0 & 1 & 0 & 0 & 0 & 0 & 1 & 0 & 0 & 1 & 0 & 0 \\
\hline Acc 13 & 0 & 1 & 0 & 0 & 0 & 1 & 0 & 0 & 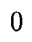 & 0 & 1 & 0 & 0 & 0 & 1 & 0 & 0 & 0 & 1 & 0 & 0 \\
\hline Acc 14 & 0 & 1 & 0 & 0 & 1 & 0 & 0 & 0 & 0 & 1 & 0 & 0 & 0 & 0 & 1 & 0 & 0 & 0 & 1 & 0 & 0 \\
\hline Acc 15 & 0 & 1 & 0 & 0 & 1 & 0 & 0 & 0 & 1 & 0 & 0 & 0 & 0 & 0 & 1 & 0 & 0 & 0 & 1 & 0 & 0 \\
\hline Acc 16 & 0 & 1 & 0 & 0 & 1 & 0 & 0 & 0 & 0 & 1 & 0 & 0 & 0 & 0 & 1 & 0 & 0 & 0 & 0 & 1 & 0 \\
\hline Acc 17 & 0 & 1 & 0 & 0 & 0 & 1 & 0 & 0 & 0 & 0 & 1 & 0 & 0 & 0 & 1 & 0 & 0 & 0 & 0 & 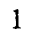 & 0 \\
\hline Acc 18 & 0 & 1 & 0 & 0 & 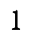 & 0 & 0 & 0 & 1 & 0 & 0 & 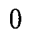 & 0 & 0 & 1 & 0 & 0 & 0 & 1 & 0 & 0 \\
\hline Acc 19 & 0 & 1 & 0 & 0 & 0 & 1 & 0 & 0 & 0 & 0 & 1 & 0 & 0 & 0 & 0 & 0 & 1 & 0 & 1 & 0 & 0 \\
\hline Acc 20 & 0 & 1 & 0 & 0 & 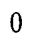 & 1 & 0 & 0 & 0 & 0 & 1 & 0 & 0 & 0 & 0 & 0 & 1 & 0 & 1 & 0 & 0 \\
\hline Acc 21 & 0 & 0 & 1 & 0 & 0 & 1 & 0 & 0 & 0 & 0 & 0 & 1 & 0 & 0 & 1 & 0 & 0 & 0 & 0 & 1 & 0 \\
\hline Acc 22 & 0 & 0 & 1 & 0 & 1 & 0 & 0 & 0 & 0 & 1 & 0 & 0 & 0 & 0 & 1 & 0 & 0 & 0 & 0 & 1 & 0 \\
\hline Acc 23 & 0 & 1 & 0 & 0 & 0 & 1 & 0 & 0 & 0 & 0 & 1 & 0 & 0 & 0 & 1 & 0 & 0 & 0 & 1 & 0 & 0 \\
\hline $\operatorname{Acc} 24$ & 0 & 0 & 1 & 0 & 1 & 0 & 0 & 0 & 1 & 0 & 0 & 0 & 0 & 0 & 1 & 0 & 0 & 0 & 1 & 0 & 0 \\
\hline Acc 25 & 0 & 1 & 0 & 0 & 0 & 1 & 0 & 0 & 0 & 0 & 1 & 0 & 0 & 0 & 1 & 0 & 0 & 0 & 0 & 1 & 0 \\
\hline $\operatorname{Acc} 26$ & 0 & 1 & 0 & 0 & 0 & 0 & 1 & 0 & 0 & 0 & 0 & 1 & 0 & 0 & 1 & 0 & 0 & 0 & 0 & 1 & 0 \\
\hline Acc 27 & 0 & 1 & 0 & 0 & 0 & 1 & 0 & 0 & 0 & 0 & 1 & 0 & 0 & 0 & 0 & 1 & 0 & 0 & 1 & 0 & 0 \\
\hline Acc 28 & 0 & 1 & 0 & 0 & 0 & 0 & 1 & 0 & 0 & 0 & 0 & 1 & 0 & 0 & 1 & 0 & 0 & 0 & 1 & 0 & 0 \\
\hline Acc 29 & 1 & 0 & 0 & 0 & 0 & 1 & 0 & 0 & 0 & 1 & 0 & 0 & 0 & 0 & 1 & 0 & 0 & 0 & 1 & 0 & 0 \\
\hline $\operatorname{Acc} 30$ & 0 & 0 & 0 & 1 & 0 & 0 & 1 & 0 & 0 & 0 & 0 & 0 & 1 & 0 & 0 & 1 & 0 & 0 & 0 & 0 & 1 \\
\hline Total & 3 & 20 & 6 & 1 & 7 & 14 & 7 & 2 & 3 & 6 & 12 & 4 & 5 & 0 & 19 & 8 & 3 & 0 & 15 & 14 & 1 \\
\hline
\end{tabular}

accessions and categorized into greenish yellow, yellow green, green, dark green and light green orange. The number of genotypes fallen under each group were 11, 11, two, five and one, respectively. Only the accession, Acc 9 could be identified for its unique attractive fruit color (light green orange). Acc 30 along with Acc 6, Acc 7, Acc 8 and Acc 12 had dark green fruits. Like fruit color, fruit shape had also shown much variability among the accessions such as round, oval, cylindrical, oblong and spindle. Acc 29 only had the characteristic tennis-ball-like round fruit and could be identified as unique marker of that accession. About 11 accessions had oval, six had cylindrical, seven had oblong and the rest five had spindle shaped fruit. Generally, growers prefer cylindrical to spindle shaped fruit with greenish color, sometimes oval fruit, so there is ample scope to be bred to fulfill consumer choice using the existing fruit variability of kakrol in Bangladesh.

The length of fruits was quite variable from short (shorter than $50 \mathrm{~mm}$ ) to very long (longer than $130 \mathrm{~mm}$ ) category. The Acc 29 and Acc 30 had the extreme value such as short and very long, respectively. Fruit length showed similar trend with fruit diameter 
i.e. most medium length fruits had medium diameter. The fruits of Acc 29 and Acc 30 possessed small and large diameter, respectively. The individual fruit weight varied from $40 \mathrm{~g}$ (Acc 29) to $625 \mathrm{~g}$ (Acc 30) and was grouped as light ( $<50 \mathrm{~g}$ ), medium (51-100 g), heavy (101-150 g) and very heavy $(>151 \mathrm{~g})$. Number of fruits per plant was classified into four classes, viz. few (23\%), moderate (47\%), many (23\%) and profuse (7\%). Acc 3 and Acc 7 yielded 61 and 62 fruits per plant, respectively. Fruit yield varied from $0.6 \mathrm{~kg}$ (Acc 15) to $32 \mathrm{~kg}$ (Acc 30). Five genotypes (Acc 5, Acc 7, Acc 8, Acc 9 and Acc 30) occupied bumper yield group, and it might be due to the combination effect of fruit number and individual fruit weight.

Nineteen accessions produced 16-30 seeds per fruit, eight had 31-45 seeds and only three (Acc 8, Acc 19 and Acc 20) contained many seeds (46-60). Consumers do not prefer the presence of large number of seeds in fruits because it decreases the fruit palatability. So breeders should develop cultivars having fruits with less number of seeds. The 20-seed weight varied from 2.7 to $32.8 \mathrm{~g}$. The lowest was in Acc 3 of Rangpur region and the highest in Acc 30 (M. cochinchinensis) collected from Thakurgaon area. Fifteen accessions had medium and 14 accessions slightly bold seeds.

In this study, the morphological and physiological variation was not much prominent among $29 M$. dioica accessions but it was remarkable between the species ( $M$. dioica and $M$. cochinchinensis). Round stem, serrated leaves, no pubescence on the leaf surface, large fruit size, bold seed, perennial growth habit and high yield seemed to be the characteristics featuring of $M$. cochinchinensis. M. dioica possessed 5-ridged stem, many stems, dented medium leaves, pubescence on leaf surface, many small to medium fruits, small seeds with annual growth habit. These characteristics might be considered as salient feature of this species. The descriptors mentioned in Table 2 are recommended to breeders to characterize germplasm or landrace collections in Bangladesh.

\section{Cluster analysis}

Cluster analysis grouped the genoytpes into two distinct classes (Fig. 1). The cultivars of $M$. dioica were clearly separated from its wild relative, $M$. cochinchinensis indicating that morpho-physiological variation could be used in classifying Momordica genus at species level. Among 29 morpho-physiological characters studied, the variation exhibited in days to flowering, stem and leaf characteristics, fruit and seed size and finally yield seemed to play major role in separating the two species. Sultana et al. (2001) also reported such separation between wild and cultivated species using morphological variation in lablab bean. Based on morphological variation, Hubbard and Garbary (2002) also classified seaweed (Codium spp.) in different subspecies using cluster analysis and principal component analysis. The dissimilarity order in the present study as observed in dissimilarity matrix (data not shown) was Acc 30>Acc 27>Acc 29>Acc $23>$ Acc $19>$ Acc 14 and likely they were grouped separately with the rest of the accessions.

The 30 accessions were divided into five distinct clusters, A, B, C, D and E, and each group contained one, two, two, one and 24 accessions, respectively (Fig. 1). Group A contained the wild accession, Acc 30. Group B contained Acc 29 and Acc 27 of which Acc 29 produced tennis ball like small fruits weighing only $40 \mathrm{~g}$. Group $\mathrm{C}$ had two accessions (Acc 19 and Acc 23) having heavy fruits with large number of seeds per fruit. Group D possessed a single accession (Acc 14) that had medium performance for most of the para- 


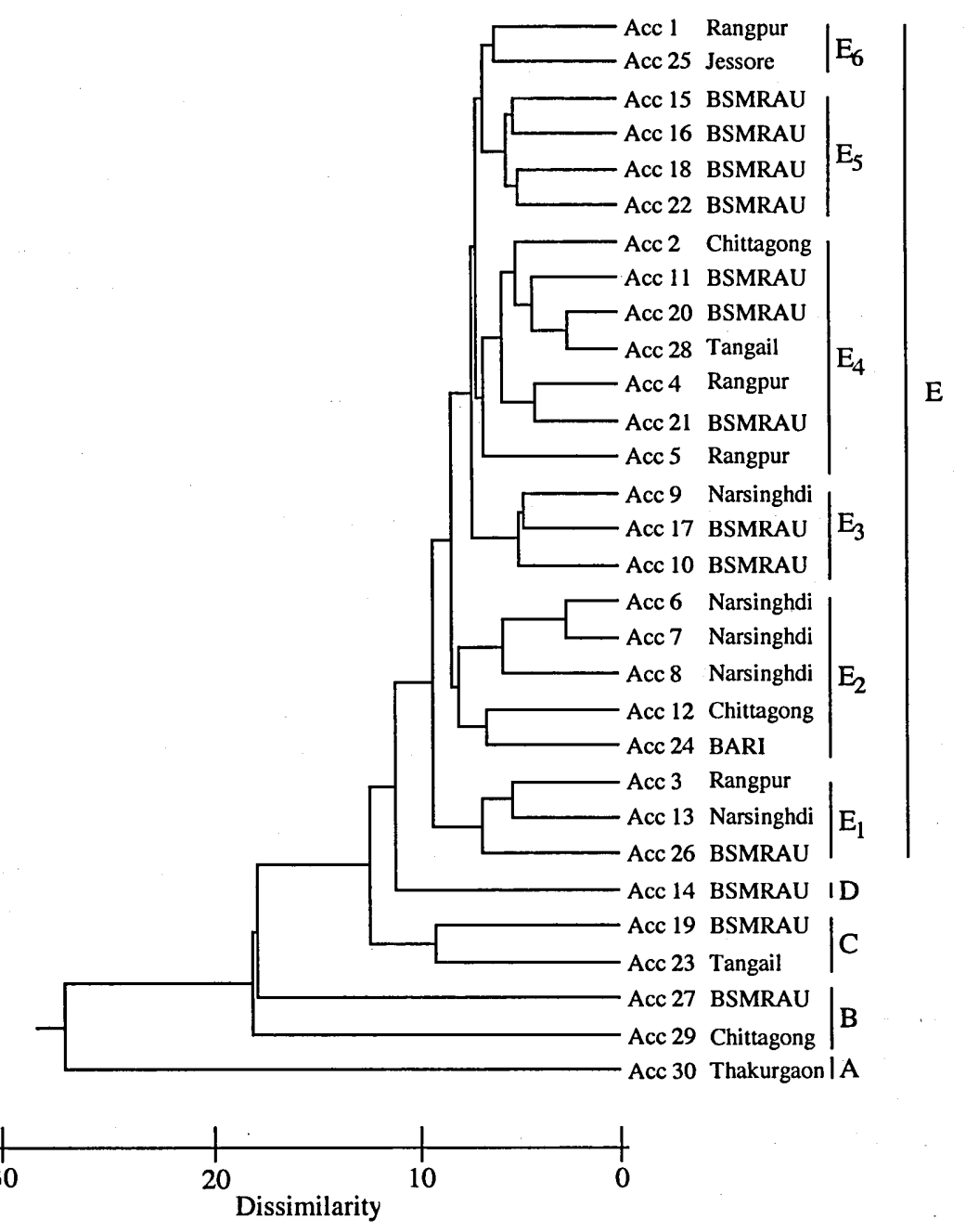

Fig. 1. Dendrogram of 30 accessions constructed by morphological and physiological characteristics using UPGMA based on Euclidean dissimilarity values.

meters with less number of fruits with less yield. The rest of the 24 accessions formed a single cluster $\mathrm{E}$. The Group $\mathrm{E}$ again divided into six sub-clusters $\mathrm{E}_{1}, \mathrm{E}_{2}, \mathrm{E}_{3}, \mathrm{E}_{4}, \mathrm{E}_{5}$ and $\mathrm{E}_{6}$. The medium variable parameters like floral structure (ovary, style and pistil), fruit shape, size (length and diameter), color, number of fruits and yield were contained in this cluster. The results obtained here may indicate that there is high heterogeneity in morphological characters within collected accessions but it is difficult to identify within species.

All the accessions were collected from different agroecological zones of Bangladesh. No particular characters or genotypes from a single location formed a single cluster. This 
may be due to free exchange of seed material among the growers of different regions, and as a consequence the character constellation, that might be associated with particular region, have not lost their individuality under human interference (Chowdhury et al., 1998). Similarly, Bhadra and Akhter (1991) in black gram, Natarajan et al. (1988) in green gram, Patel et al. (1989) in sunflower, Rahman et al. (2001) in Capsicum, Rasul and Okubo (2002) in kakrol found no relationship with geographical isolation and genetic diversity of the crop. Subcluster $\mathrm{E}_{5}$ contained four accessions (Acc 15, Acc 16, Acc 18 and Acc 22) of the same place (bred at BSMRAU, Gazipur) having closer and lesser dissimilarity value (ranging from 9.0 to 10.0 for all possible combination).

\section{REFERENCES}

Bhadra, S. K. and M. I. Akhtar 1991 Genetic divergence for yield and its morphological components of mungbean (Vigna radiata (L) Wilczek). SABRAO Journal, 23: 127-136

Brown, R. N. and J. R. Myers 2002 A genetic map of squash (Cucurbita $s p$ ) with randomly amplified polymorphic markers and morphological markers. J. Amer. Soc. Hort. Sci., 127: 568-575

Bose T. K. and M. J. Som 1986 Vegetable Crops in India. Naya Prakash, Calcutta, India

Chowdhury, M. A. Z., M. F. U. Mia, M. S. Alam and E. H. Chaudhury 1998 Genetic diversity in soybean (Glycine max. L Merril). Bangladesh J. Pl. Breed. Genet., 7: 55-58

Felsenstein, J. 1995 Phylogeny Inferance Package (PHYLIP) Version 3.57c. University of Washington, Seattle, USA.

Grant, M. L., D. M. Miller and A. Culham 2001 Genetic and morphological analysis of the origin and identity of perennial Lavatera (Malvaceae) cultivars. J. Amer. Soc. Hort. Sci., 125: 593-598

Hossain, S. M. M., M. Tarik and A. K. M. S. R. Mollick 1992 Evaluation of teasle gourd genotypes in Bangladesh. Edited by A. K. M. A. Hosssain, published by HRC, Bangladesh Agricultural Research Institute, Gazipur 1701, Bangladesh.

Hubbard, C. B. and D. J. Garbary 2002 Morphological variation of Codium fragile (Chlorophyta) in Eastern Canada. Botanica Marina., 45: 476-486

Hussain, M. A. and M. M. Rashid 1974 Floral biology of kakrol. Bangladesh Hort., 2: 1-4

Masud, M. A. T, M. A. Z Chowdhury, M. A. Hossain and S. M. M. Hossain 1995 Multivariate analysis in pumpkin (Cucurbita moschata Duch ex poir). Bangladesh J. Pl. Breed. Genet., 8: 45-50

Natarajan, C., K. Thiagarajan and R. Rathanaswamy 1988 Association and genetic diversity studies in green gram. Madras Agric. J., 75: 238-245

Patel, M. Z., M. V. Reddy, B. S. Rana and B. Reddy 1989 Genetic divergence in safflower (Carthamus tinctorious L). Indian J. Genet., 49: 113-118

Rahman, M. M., M. G. Rasul, M. S. Islam, N. Sultana and M. A. Hossain 2001 Genetic diversity in Capsicum sp. Bangladesh J. Pl. Breed. Genet., 14: 7-11

Rashid, M. M. 1993 Sabjee Biggan (In Bengali). Bangla Academy. Dhaka. Bangladesh.

Rasul, M. G. and H. Okubo 2002 Genetic diversity in teasle gourd (Momordica dioica Roxb.). Bangladesh J. Pl. Breed. Genet., 15: 7-15

Sokal, R. R. and C. D. Michener 1958 A statistical method for evaluating systemic relationships. Univ. Kansas Sci. Bull., 38: 1409-1438

Sultana, N., Y. Ozaki and H. Okubo 2001 Morphological and physiological variation in lablab bean (Lablab purpureus (L) Sweet.). J. Fac. Agr., Kyushu Univ., 45: 465-472

Trivedi, R. N. and R. P. Roy 1972 Cytological studies in some species of Momordica. Genetica, 43: $282-291$ 